\title{
The Fanconi anemia DNA damage repair pathway in the spotlight for germline predisposition to colorectal cancer
}

\author{
Clara Esteban-Jurado ${ }^{1}$, Sebastià Franch-Expósito ${ }^{1}$, Jenifer Muñoz ${ }^{1}$, Teresa Ocaña ${ }^{1}$, Sabela Carballal ${ }^{1}$,
} Maria López-Cerón ${ }^{1}$, Miriam Cuatrecasas ${ }^{2}$, Maria Vila-Casadesús ${ }^{3}$, Juan José Lozano ${ }^{3}$, Enric Serra ${ }^{4}$, Sergi Beltran ${ }^{4}$, The EPICOLON Consortium, Alejandro Brea-Fernández ${ }^{5}$, Clara Ruiz-Ponte ${ }^{5}$, Antoni Castells ${ }^{1}$, Luis Bujanda ${ }^{6}$, Pilar Garre ${ }^{7}$, Trinidad Caldés ${ }^{7}$, Joaquín Cubiella ${ }^{8}$, Francesc Balaguer ${ }^{1}$ and Sergi Castellví-Bel ${ }^{\star, 1}$

Colorectal cancer (CRC) is one of the most common neoplasms in the world. Fanconi anemia (FA) is a very rare genetic disease causing bone marrow failure, congenital growth abnormalities and cancer predisposition. The comprehensive FA DNA damage repair pathway requires the collaboration of 53 proteins and it is necessary to restore genome integrity by efficiently repairing damaged DNA. A link between FA genes in breast and ovarian cancer germline predisposition has been previously suggested. We selected 74 CRC patients from 40 unrelated Spanish families with strong CRC aggregation compatible with an autosomal dominant pattern of inheritance and without mutations in known hereditary CRC genes and performed germline DNA wholeexome sequencing with the aim of finding new candidate germline predisposition variants. After sequencing and data analysis, variant prioritization selected only those very rare alterations, producing a putative loss of function and located in genes with a role compatible with cancer. We detected an enrichment for variants in FA DNA damage repair pathway genes in our familial CRC cohort as 6 families carried heterozygous, rare, potentially pathogenic variants located in BRCA2/FANCD1, BRIP1/FANCJ, FANCC, FANCE and REV3LPOLZ. In conclusion, the FA DNA damage repair pathway may play an important role in the inherited predisposition to CRC.

European Journal of Human Genetics (2016) 24, 1501-1505; doi:10.1038/ejhg.2016.44; published online 11 May 2016

\section{INTRODUCTION}

Colorectal cancer (CRC) is the third most frequent neoplasm in the world and its average lifetime risk in the general population is $~ 5 \%$. ${ }^{1}$ There is some degree of familial aggregation in up to $35 \%$ of CRC patients, but the majority of the underlying germline predisposition factors remain still unidentified. The Mendelian CRC syndromes, with Lynch syndrome and familial adenomatous polyposis being the most common, correspond to $5 \%$ of total CRC cases and are mainly due to germline mutations in APC, MUTYH and the mismatch repair genes (ie, MLH1, MSH2, MSH6, PMS2). Recently, next-generation sequencing efforts in familial CRC have identified additional causative germline mutations in genes such as POLE, POLD1 and NTHL1..$^{2-4}$

Fanconi anemia (FA) is a very rare genetic disease with an incidence of 1-3 per 500000 births and it causes bone marrow failure, congenital growth abnormalities and cancer predisposition. FA patients have chromosome fragility and hypersensitivity to drugs that induce DNA interstrand crosslinks (ICLs). ${ }^{5}$ It corresponds to an autosomal recessive condition and it has been associated with germline mutations in 18 FA genes. Among them, monoallelic mutations in
FANCD1/BRCA2, FANCJ/BRIP1, FANCN/PALB2 and FANCC have also been linked to breast and ovarian cancer genetic predisposition. ${ }^{6,7}$ The comprehensive FA DNA damage repair pathway requires the collaboration of 53 proteins and it is necessary to restore genome integrity by efficiently repairing damaged DNA, especially ICLs (Figure 1). ICLs affect both DNA strands impeding transcription and replication-fork progression and also complicating correct DNA repair as there is no unaffected template available. ${ }^{8}$ Besides the link between FA genes and breast and ovarian cancer, some other genes not contributing to FA but part of the FA DNA damage repair pathway have additionally been involved in the same cancer predisposition and include BRCA1, RAD51C and FANCM. ${ }^{9}$ Very recently, mutations in some FA DNA damage repair pathway genes have also been postulated to be the germline triggers in familial CRC cases, including BRCA2, ${ }^{10,11}$ FAN $^{12}$ and BLM. ${ }^{13}$

\section{MATERIALS AND METHODS}

We selected 74 CRC probands from 40 unrelated Spanish families (4 affected relatives from 1 family, 3 affected relatives from 8 families, 2 affected relatives

\footnotetext{
${ }^{1}$ Gastroenterology Department, Hospital Clínic, Institut d'Investigacions Biomèdiques August Pi i Sunyer (IDIBAPS), Centro de Investigación Biomédica en Red de Enfermedades Hepáticas y Digestivas (CIBEREHD), University of Barcelona, Barcelona, Spain; ${ }^{2}$ Department of Pathology, Hospital Clinic, Barcelona, Spain; ${ }^{3}$ Bioinformatics Platform, Centro de Investigación Biomédica en Red de Enfermedades Hepáticas y Digestivas (CIBEREHD), Barcelona, Spain; ${ }^{4}$ Centre Nacional d’Anàlisi Genòmica (CNAG), Parc Científic de Barcelona, Barcelona, Spain; ${ }^{5}$ Galician Public Foundation of Genomic Medicine (FPGMX), Centro de Investigación Biomédica en Red de Enfermedades Raras (CIBERER), Genomics Medicine Group, Hospital Clínico, University of Santiago de Compostela, Santiago de Compostela, Spain; ${ }^{6}$ Gastroenterology Department, Hospital Donostia-Instituto Biodonostia, Centro de Investigación Biomédica en Red de Enfermedades Hepáticas y Digestivas (CIBEREHD), Country University (UPV/EHU), San Sebastián, Spain; ${ }^{7}$ Molecular Oncology Laboratory, Hospital Clínico San Carlos, Instituto de Investigación Sanitaria del Hospital Clínico San Carlos (IdISSC), Madrid, Spain; ${ }^{8}$ Gastroenterology Department, Complexo Hospitalario Universitario de Ourense, Instituto de Investigación Biomédica Ourense, Pontevedra y Vigo, Ourense, Spain

*Correspondence: Dr S Castellví-Bel, Genetic Predisposition to Colorectal Cancer Group, IDIBAPS, Centre Esther Koplowitz (CEK), Rosselló 153 planta 4, 08036 Barcelona, Catalonia, Spain. Tel: +34 932275400 ext. 4183/2915; E-mail: sbel@clinic.ub.es

Received 16 November 2015; revised 29 March 2016; accepted 7 April 2016; published online 11 May 2016
} 


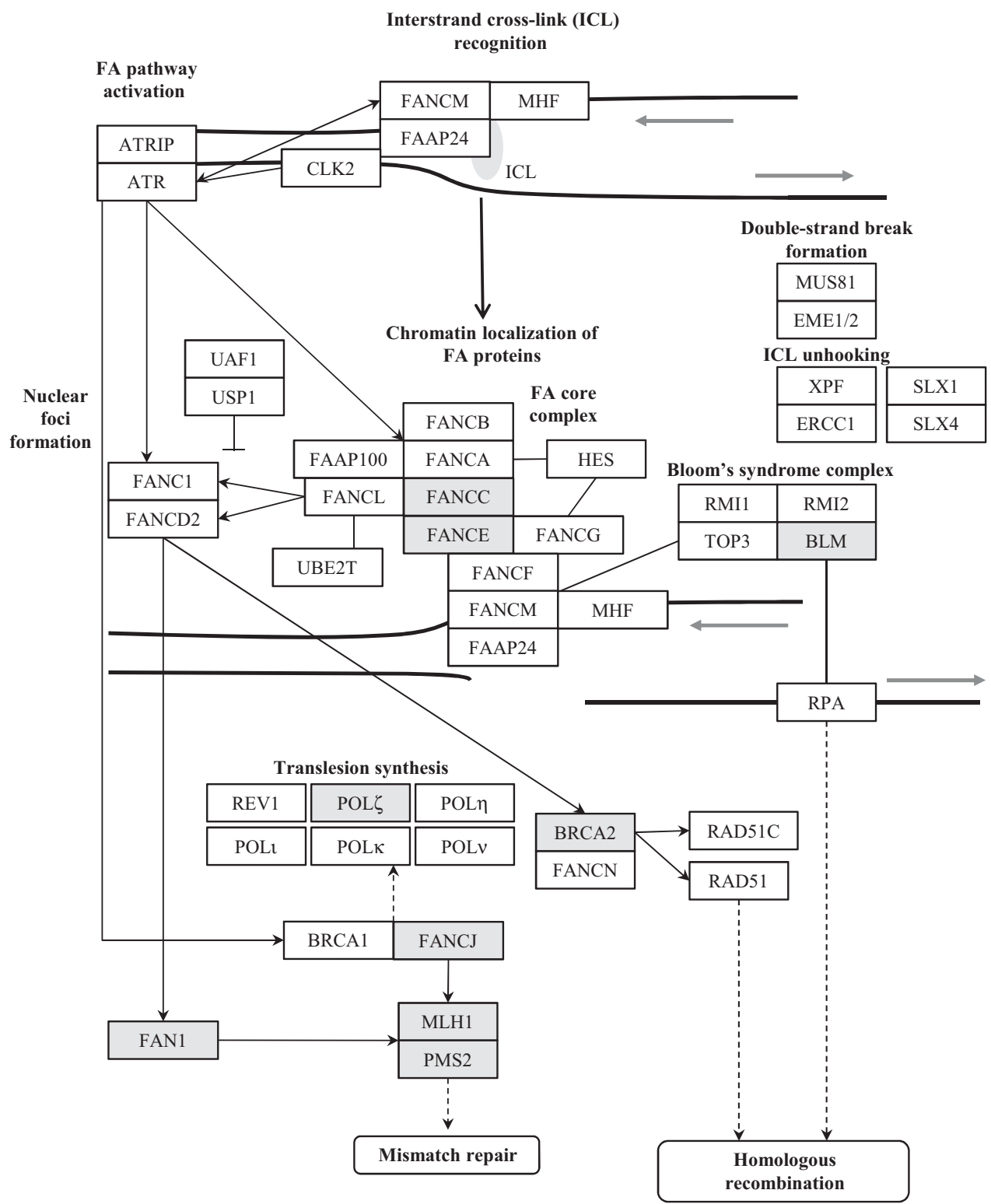

Figure 1 The Fanconi anemia (FA) DNA damage repair pathway. Genes linked to colorectal cancer (CRC) predisposition by the present report and previous evidence are shaded in gray (adapted from KEGG database, http://www.genome.jp/kegg/pathway.html).

from 15 families and 16 CRC unrelated patients) with strong CRC aggregation compatible with an autosomal dominant pattern of inheritance and without point mutations or large rearrangements in the most common hereditary CRC genes (APC, MUTYH and mismatch repair genes). Families were selected based on the following criteria: $\geq 3$ relatives with $\mathrm{CRC}, \geq 2$ consecutive affected generations and at least one CRC diagnosed before the age of 60 years. This study was approved by the institutional ethics committee of each participating hospital and written informed consent was obtained at CRC diagnosis.

Sequencing, raw data analysis and variant filtering was performed as previously described for a subset of 42 patients. ${ }^{14}$ In this regard, it should be noted that this previous cohort was completed with 31 additional new CRC patients, corresponding to 11 new families and 5 new cases in previously analyzed families, totaling $74 \mathrm{CRC}$ probands from 40 families. Briefly, germline DNA whole-exome sequencing (WES) used the HiSeq2000 platform (Illumina, San Diego, CA, USA) and SureSelectXT Human All Exon for exon enrichment V4 (Agilent, Santa Clara, CA, USA). Mean coverage was $>95 \times$ in all samples and 51 Megabases was the target size that required $\sim 4$ Gigabytes of sequencing per sample. After sequencing and data analysis, variant prioritization selected only those very rare alterations $(0-0.1 \%)$, shared by individuals sequenced from the same family, producing a putative loss of function and located in genes with a role compatible with cancer (Supplementary Table 1). Variants were validated by Sanger sequencing (GATC Biotech, Köln, Germany) and segregation analysis of the prioritized variants was performed in additional affected family members (CRC and advanced adenoma) when constitutive DNA was available. Genetic variants have been submitted to the ClinVar database (http://www.ncbi.nlm. nih.gov/clinvar/; accession numbers SCV000262600, SCV000262601, SCV000262602, SCV000262603, SCV000262604 and SCV000262605). In addition, somatic loss of heterozygosity ( $\mathrm{LOH}$ ) was studied by Sanger sequencing or microsatellites in tumor DNA of patients (one per family) carrying the selected variants when possible. DNA was extracted from a percentage of tumor cells of $70-80 \%$ in most cases.

\section{RESULTS AND DISCUSSION}

The aim of our study was to find candidate germline predisposition variants by performing exome sequencing in a cohort of familial CRC 
patients compatible with an autosomal dominant inheritance and without an alteration in the previously known hereditary CRC genes in order to facilitate genetic counseling and correctly address prevention strategies. Our preliminary results for a subset of CRC patients were previously published ${ }^{14}$ but additional family members and new families were whole-exome sequenced more recently and the present report corresponds to the analysis of the complete cohort of 74 samples.

Interestingly, our data revealed heterozygous, rare, potentially pathogenic variants in six families located in genes belonging to the FA DNA damage repair pathway including BRCA2/FANCD1, BRIP1/ FANCJ, FANCC, FANCE and REV3L/POLZ after data analysis and variant prioritization (Table 1 and Figure 2). All six prioritized variants were validated by Sanger sequencing (Supplementary Figure 1) and segregation analysis was correct in family members (CRC and advanced adenoma). It is also interesting to point out that among the 1-57 variants identified by WES that remained after filtering in each family, the genetic variants finally prioritized corresponded to the best candidate for genetic predisposition to CRC (not reported or with a very low frequency $<0.01 \%$ in external control exome data sets including a Spanish database, potential loss-of-function variant affecting residues highly conserved in evolution, previous implication with cancer predisposition and correct segregation). It is important to highlight that three of the variants corresponded to frameshift alterations and three to missense variants. The former are expected to truncate the protein and, therefore, are likely pathogenic, whereas for the latter pathogenicity is plausible but has not been proven with functional studies. On the other hand, the identified missense variants comply with several criteria to be potentially deleterious and two of them (those in BRCA2 and FANCE) that fall in protein domains where pathogenic mutations have been previously reported in FA or familial breast and ovarian cancer patients in the ClinVar database or the FA mutation database (http://www.rockefeller.edu/fanconi/). In addition, when comparing with a publically available genetic variation control data set (Exome Aggregation Consortium), we detected a clear enrichment for putative loss-of-function variants in these FA DNA damage repair pathway genes in our familial CRC cohort, when considering nonsense, canonical splice site, frameshift and missense variants with a likely pathogenicity prediction (CADD > 15, Combined Annotation Dependent Depletion, CADD, http://cadd.gs.washington.edu/) and a genotype frequency $<0.01 \%$ $\left(6 / 40,15 \%\right.$ vs 2546/60 706, 4.2\%; $\chi^{2}$ test, uncorrected for multiple testing $P$-value $=0.0025)$, or only nonsense, canonical splice site and frameshift with a genotype frequency $<0.01 \%(3 / 40,7.5 \%$ vs $306-$ $160706,0.5 \%$; $\chi^{2}$ test, uncorrected for multiple testing $P$-value $<0.0001)$. It is also worth mentioning that in 5 of the 6 families carrying the reported variants, other neoplasms besides CRC were present with an age of onset $<60$ years, including breast cancer, endometrial cancer, prostate cancer, lung cancer, leukemia and gastric cancer. No relevant clinical or histopathological characteristics were detected among variant carriers, although a small sample size could be precluding the detection of such correlation.

Taking into account our results and previous evidence, ${ }^{10-13}$ our report draws the attention to the fact that the FA DNA damage

Table 1 Description of the 6 genetic variants belonging to the Fanconi anemia DNA damage repair pathway detected in a cohort of 40 Spanish colorectal cancer families

\begin{tabular}{|c|c|c|c|c|c|c|c|c|c|c|}
\hline Family & Gene & RefSeq & Genetic variant & $\begin{array}{l}\text { Genotype fre- } \\
\text { quency (ExAC, } \\
\text { EVS, CSVS) }\end{array}$ & $\begin{array}{l}\text { In } \\
\text { silico }\end{array}$ & Family phenotype (age <60) & $\begin{array}{l}\text { Cancer- } \\
\text { AA car- } \\
\text { riers }\end{array}$ & $\mathrm{LOH}$ & $\begin{array}{l}\text { Domain/ } \\
\text { region }\end{array}$ & $O M I M$ \\
\hline FAM6 & $\begin{array}{l}\text { BRCA2/ } \\
\text { FANCD1 }\end{array}$ & NM_000059.3 & $\begin{array}{l}\text { c. } 7759 C>T p . \\
(L 2587 F)\end{array}$ & $\begin{array}{l}5 / 60676 \\
0 / 6503 \\
0 / 572\end{array}$ & $5 / 5$ & $\mathrm{CRC}$, gastric & $2 / 2$ & $\mathrm{INC}^{\mathrm{a}}$ & $\begin{array}{l}\text { Interaction } \\
\text { with DSS1 }\end{array}$ & $\begin{array}{l}\text { Breast and ovarian } \\
\text { cancer, FA }\end{array}$ \\
\hline FAM20 & $\begin{array}{l}\text { BRCA2/ } \\
\text { FANCD1 }\end{array}$ & NM_000059.3 & $\begin{array}{l}\text { c.4963delT p. } \\
\left(Y 1655 f{ }^{*} 15\right)\end{array}$ & $\begin{array}{l}0 / 60706 \\
0 / 6503 \\
0 / 572\end{array}$ & FS & $\begin{array}{l}\text { CRC, breast cancer, endometrial } \\
\text { cancer, prostate cancer, lung } \\
\text { cancer, leukemia }\end{array}$ & $2 / 2$ & $\mathrm{INC}^{\mathrm{a}}$ & - & $\begin{array}{l}\text { Breast and ovarian } \\
\text { cancer, FA }\end{array}$ \\
\hline H463 & $\begin{array}{l}\text { BRIP1/ } \\
\text { FANCJ }\end{array}$ & NM_032043.2 & $\begin{array}{l}\text { c.1702_1703delAA } \\
\text { p. }(\mathrm{N} 568 \mathrm{fs} * 9)^{\mathrm{b}}\end{array}$ & $\begin{array}{l}0 / 60706 \\
0 / 6503 \\
0 / 572\end{array}$ & FS & $\mathrm{CRC}$, gastric cancer & $1 / 1$ & $\mathrm{INC}^{\mathrm{a}}$ & - & $\begin{array}{l}\text { Breast and ovarian } \\
\text { cancer, Spanish FA } \\
\text { J familyc }\end{array}$ \\
\hline FAMN4 & FANCC & NM_000136.2 & $\begin{array}{l}\text { c.591_592dupC p. } \\
(\text { L199fs*12) }\end{array}$ & $\begin{array}{l}0 / 60706 \\
0 / 6503 \\
0 / 572\end{array}$ & FS & CRC, adenomas & $2 / 2$ & $\mathrm{~N}$ & - & $\begin{array}{l}\text { Breast cancer, pan- } \\
\text { creatic cancer, FA }\end{array}$ \\
\hline FAM40 & FANCE & NM_021922.2 & $\begin{array}{l}\text { C. } 598 C>T p . \\
(R 200 C)\end{array}$ & $\begin{array}{l}0 / 60706 \\
1 / 6503 \\
0 / 572\end{array}$ & $5 / 5$ & CRC, breast cancer & $2 / 2$ & NA & $\begin{array}{l}\text { Interaction } \\
\text { with } \\
\text { FANCC }\end{array}$ & $\begin{array}{l}\mathrm{FA} \text {, esophageal and } \\
\text { gastric cancer }\end{array}$ \\
\hline FAM3 & $\begin{array}{l}\text { REV3L/ } \\
\text { POLZ }\end{array}$ & NM_002912.4 & $\begin{array}{l}\text { c. } 559 A>T p . \\
(R 187 W)\end{array}$ & $\begin{array}{l}0 / 60706 \\
0 / 6503 \\
0 / 572\end{array}$ & $5 / 5$ & CRC, prostate cancer, adenomas & $4 / 4$ & Y & - & $\begin{array}{l}\text { Lung cancer, chro- } \\
\text { mosomal instability }\end{array}$ \\
\hline
\end{tabular}

Abbreviations: AA, advanced adenoma; CRC, colorectal cancer; FA, Fanconi anemia; FS, frameshift; INC, inconclusive; LD, linkage disequilibrium; LOH, loss of heterozygosity; N, no; NA, not available; RefSeq, reference sequence; Seg, segregation; $Y$, yes.

Genotype frequency: presence or absence in external control exome data sets (ExAC (exome aggregation consortium), EVS (exome variant server) and CSVS (CIBERER Spanish variant server)). In silico: number of deleterious predictions by bioinformatics tools used (CADD, PolyPhen, SIFT, PhyloP and LRT).

Cancer-AA carriers: number of cancer/advanced adenoma cases within the family that carry the variant.

LOH: depletion of the wild-type allele in tumor DNA in comparison with the germline.

Domain/region: the protein domain or region where the variant is located.

OMIM: OMIM database information including previous hereditary cancer involvement.

aMinimal LOH was observed.

bPreviously reported in Esteban-Jurado et al. ${ }^{14}$

cSame variant in our CRC cohort and in the Spanish FA J family. 
repair pathway may play an important role in the inherited predisposition to CRC. It is also important to highlight that pleiotropy is becoming important in germline predisposition to cancer as a higher number of genes may be involved in the genetic predisposition to a broader spectrum of neoplasms, as evidenced by our results and others. ${ }^{11}$ Finally, it could be hypothesized that defects in the FA DNA
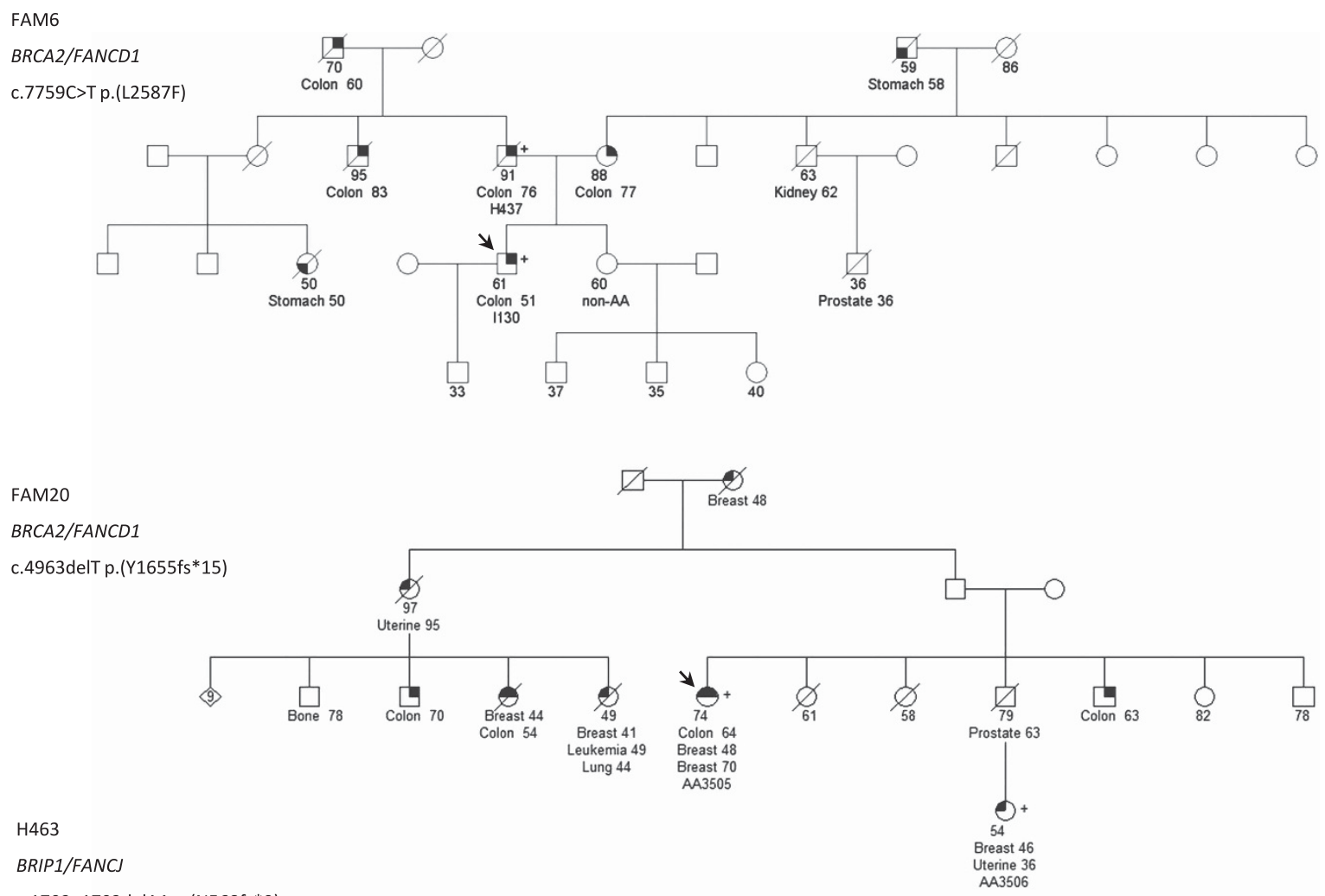

C.1702_1703delAA p.(N568fs*9)
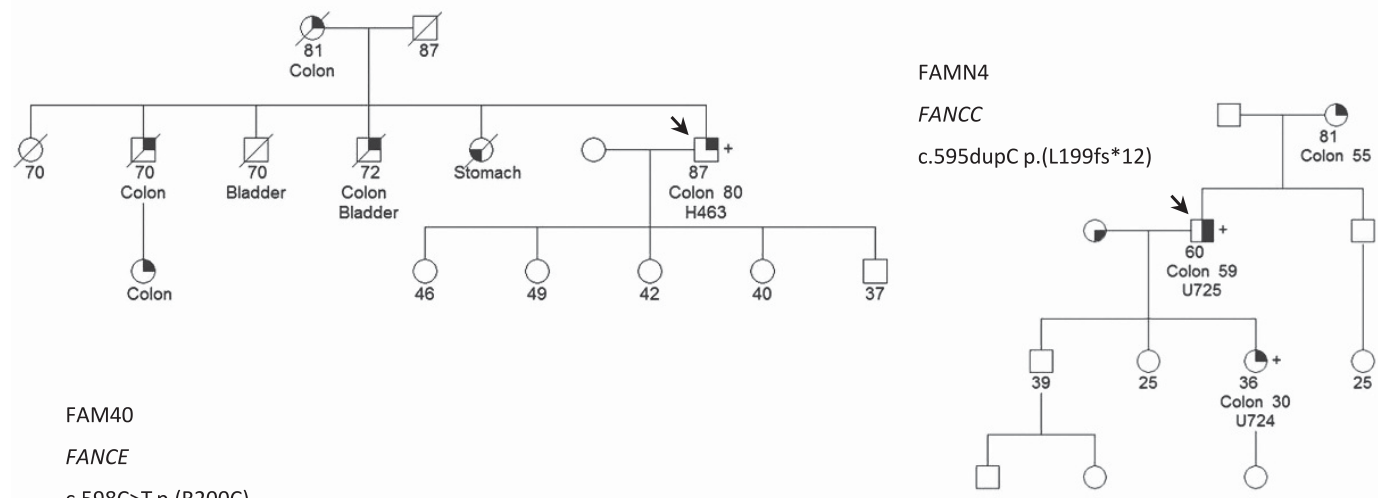

FANCE

C.598C $>$ T p. $($ R200C)
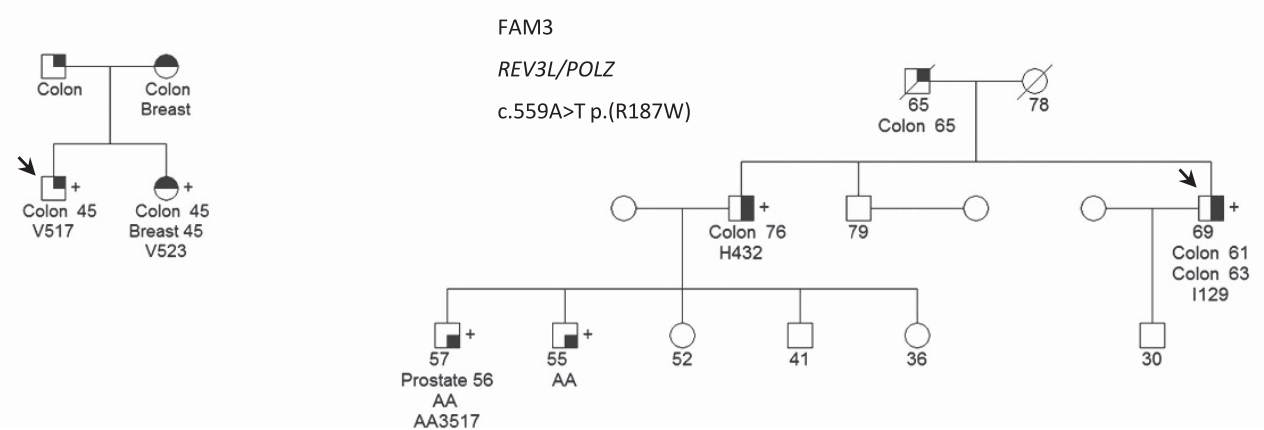

Figure 2 Pedigrees from families FAM3, FAMN4, FAM6, FAM20, FAM40 and H463 are shown. Filled symbol indicate affected for CRC (upper right quarter) adenoma/s (lower right quarter), stomach cancer (lower left quarter) and breast cancer (upper left quarter). Colon, breast, stomach, lung, prostate, kidney, uterine, leukemia and bladder refer to the type of cancer. AA/non-AA, advanced adenoma/nonadvanced adenoma; +, variant carrier. Index cases are indicated with an arrow. 
damage repair pathway would affect correct homologous recombination and contribute to genome instability. However, the contribution to CRC predisposition of genetic variants in this pathway needs further investigation and collaborative efforts should be made in order to fully characterize it. If this involvement is further confirmed in additional familial CRC cohorts, it would become very relevant regarding the molecular genetic diagnosis for the hereditary forms of this neoplasm.

\section{CONFLICT OF INTEREST}

The authors declare no conflict of interest.

\section{ACKNOWLEDGEMENTS}

We are sincerely grateful to the patients and their families for their participation. CE-J and JM are supported by a contract from CIBERehd. MV-C is supported by Ministerio de Educación, Cultura y Deporte (FPU12/05138). PG is supported by a contract from the Fondo de Investigación Sanitaria (JR13/00013). CIBERehd and CIBERER are funded by the Instituto de Salud Carlos III. This work was supported by grants from the Fondo de Investigación Sanitaria/FEDER (13/02588, 14/00173, 14/00230), the Ministerio de Economía y Competitividad (SAF2014-54453-R), Fundación Científica de la Asociación Española contra el Cáncer (GCB13131592CAST), COST Action BM1206, Beca Grupo de Trabajo ‘Oncología’ AEG (Asociación Española de Gastroenterología) and Agència de Gestió d'Ajuts Universitaris i de Recerca (Generalitat de Catalunya, 2014SGR255, 2014SGR135). We thank the Centre Nacional d'Anàlisi Genòmica and the Biobank of Hospital Clínic-IDIBAPS, Barcelona, for technical help. The work was carried out (in part) at the Esther Koplowitz Centre, Barcelona.

1 Ferlay J, Shin HR, Bray F, Forman D, Mathers C, Parkin DM: Estimates of worldwide burden of cancer in 2008: GLOBOCAN 2008. Int J Cancer 2010; 127: 2893-2917.

2 Short E, Thomas LE, Hurley J, Jose S, Sampson JR: Inherited predisposition to colorectal cancer: towards a more complete picture. J Med Genet 2015; 52: 791-796.
3 Palles C, Cazier JB, Howarth KM et al: Germline mutations affecting the proofreading domains of POLE and POLD1 predispose to colorectal adenomas and carcinomas. Nat Genet 2013; 45: 136-144.

4 Weren RD, Ligtenberg MJ, Kets CM et al: A germline homozygous mutation in the baseexcision repair gene NTHL1 causes adenomatous polyposis and colorectal cancer. Nat Genet 2015; 47: 668-671.

5 Kitao H, Takata M: Fanconi anemia: a disorder defective in the DNA damage response. Int J Hematol 2011; 93: 417-424.

6 Thompson ER, Doyle MA, Ryland GL et al: Exome sequencing identifies rare deleterious mutations in DNA repair genes FANCC and BLM as potential breast cancer susceptibility alleles. PLoS Genet 2012; 8: e1002894.

7 Bogliolo M, Surrallés J: Fanconi anemia: a model disease for studies on human genetics and advanced therapeutics. Curr Opin Genet Dev 2015; 33: 32-40.

8 Walden H, Deans AJ: The Fanconi anemia DNA repair pathway: structural and functional insights into a complex disorder. Annu Rev Biophys 2014; 43: 257-278.

9 Economopoulou P, Dimitriadis G, Psyrri A: Beyond BRCA: new hereditary breast cancer susceptibility genes. Cancer Treat Rev 2015; 41: 1-8.

10 Garre P, Martín L, Sanz J et al: BRCA2 gene: a candidate for clinical testing in familial colorectal cancer type X. Clin Genet 2015; 87: 582-587.

11 Yurgelun MB, Allen B, Kaldate RR et al: Identification of a variety of mutations in cancer predisposition genes in patients with suspected Lynch syndrome. Gastroenterology 2015; 149: 604-613.

12 Seguí N, Mina LB, Lázaro C et al: Germline mutations in FAN1 cause hereditary colorectal cancer by impairing DNA repair. Gastroenterology 2015; 149: 563-566.

13 de Voer RM, Hahn MM, Mensenkamp AR et al: Deleterious germline BLM mutations and the risk for early-onset colorectal cancer. Sci Rep 2015; 5: 14060.

14 Esteban-Jurado C, Vila-Casadesús M, Garre P et al: Whole-exome sequencing identifies rare pathogenic variants in new predisposition genes for familial colorectal cancer. Genet Med 2015; 17: 131-142.

(1) $(9)$ This work is licensed under a Creative Commons Attribution-NonCommercial-NoDerivs 4.0 International License. The images or other third party material in this article are included in the article's Creative Commons license, unless indicated otherwise in the credit line; if the material is not included under the Creative Commons license, users will need to obtain permission from the license holder to reproduce the material. To view a copy of this license, visit http://creativecommons.org/licenses/by-nc-nd/4.0/

Supplementary Information accompanies this paper on European Journal of Human Genetics website (http://www.nature.com/ejhg) 